\title{
THE REACTION OF L-HISTIDINE WITH ZIRCONIUM(IV) OXYNITRATE HYDRATE
}

\author{
de Menezes, A. S.*; Melo, E. B. \\ Departamento de Física, CCET, Universidade Federal do Maranhão, São Luís, MA, Brazil \\ *almez13@yahoo.com.br
}

\begin{abstract}
The role of L-histidine in the biological activity of natural peptides is being studied for several years. Due to the presence of imidazole ring, the histidine presents a high affinity for metal ions and these ions play a role in several different biological processes. Histidine has three coordination sites and might act either as a monodentate, bidentate or tridentate ligand. In this work the L-histidine was mixed to zirconium(IV) oxynitrate hydrate ( $\mathrm{ZrO}(-$ $\left.\mathrm{NO}_{3}\right)_{2} \times \mathrm{xH}_{2} \mathrm{O}$ ) to produce the complexation of the L-histidine with the $\mathrm{ZrO}^{2+}$ ion. For that, 1 mol of zirconium(IV) oxynitrate hydrate was added to an aqueous solution containing 2 mols of L-Histidine. However, the solution became a white hydrogel and that was an unexpect result for us at that time. Then, the hydrogel solution was centrifuged to separate the gel from the solution. The gel was dried and submitted to powder X-ray diffraction measurements that showed an amorphous pattern but it can be identified as zirconium hydroxide $\left(\mathrm{ZrO}(\mathrm{OH})_{2}\right)$. The heating of zirconium hydroxide up to $600^{\circ} \mathrm{C}$ produced nanocrystalline tetragonal and monoclinic $\mathrm{ZrO}_{2}$, being the tetragonal the higher amount phase. To know what is left in the solution, it was placed at rest at a temperature of $30^{\circ} \mathrm{C}$ to promote the evaporation of the water and to crystalize the solute. The obtained polycrystalline material was submitted to powder X-ray diffraction measurements but the obtained pattern was not indexed in comparison with the patterns of the ICDD and CCDC structural data banks.

Financial support: CNPq and FAPEMA.
\end{abstract}

